\title{
The reactivation of associated information affects source monitoring
}

\author{
B. Hunter Ball • Richard L. Marsh • J. Thadeus Meeks • \\ Jason L. Hicks
}

Published online: 1 February 2011

(C) Psychonomic Society, Inc. 2011

\begin{abstract}
In this study, we examined a source-monitoring phenomenon that arises from reactivated related information from the study phase. Three experiments showed that source attributions for target events were influenced not only by the target item itself, but also by studied information about related items. In Experiment 1, source memory for target items that have a high forward association value to a single related study item (e.g., credit) were affected by the source of the associated information (e.g., card), so that memory performance was better when associated items were presented in the same source rather than a different source. A similar effect occurred with bidirectional associates (Exp. 2), as well as with synonymous pairs of words (Exp. 3). We argue that the source information of the reactivated material can be commingled with information about a candidate during a source judgment at retrieval and thereby can affect performance.
\end{abstract}

Keywords Source monitoring - Associative strength .

Reactivation $\cdot$ Partial information

The source-monitoring framework specifies an elaborate and rich set of cognitive principles by which the contextual details of a memory trace are established, retrieved, and

\footnotetext{
B. Hunter Ball $\cdot$ R. L. Marsh

Department of Psychology, University of Georgia,

Athens, GA, USA

J. Thadeus Meeks $(\square)$

Department of Psychology,

Southern Illinois University Edwardsville,

Edwardsville, IL 62025-1121, USA

e-mail: jmeeks@siue.edu

J. L. Hicks

Department of Psychology, Louisiana State University,

Baton Rouge, LA, USA

evaluated (Johnson, Hashtroudi, \& Lindsay, 1993; Mitchell \& Johnson, 2000, 2009). Perhaps at the very core of this framework is the idea that people retrieve and evaluate the qualitative characteristics of a memory, such as the perceptual detail, any emotional reactions, the cognitive operations that came from elaborating or organizing, the spatiotemporal details, and so forth (Johnson, Foley, Suengas \& Raye 1988; Johnson \& Raye, 1981). In a typical source-monitoring judgment, the amount and quality of these details are judged against an average or otherwise expected amount of detail for a given type of memory. For example, experienced events should have greater amounts of perceptual and spatiotemporal detail as compared with memories of imagined or planned events, and the average amount and clarity expected for these details will depend on the judged age of the memory. Above and beyond the type of information that is retrieved, source-monitoring decision criteria are flexible and depend on the mental agenda at the time a source judgment is rendered. Many judgments are made heuristically and without a great deal of cognitive effort, whereas others are made more systematically, when the decision is important enough to apply plausibility checks and/or the recruitment of supporting information from memory.

According to this framework, source-monitoring errors typically occur when the qualitative characteristics retrieved are evaluated as being more like the average characteristics of a competing but incorrect source. For example, memories of planned or imagined events can be quite detailed and may include perceptual-like details, in which case they might mistakenly be labeled as perceived, or likewise, an experienced event that lacks many perceptual details might mistakenly be labeled as not perceived (e.g., Johnson, Foley, \& Leach, 1988). In general, source-monitoring errors are greater the more similar the competing sources are to one 
another. For example, two female sources will be confused more often than a male versus a female source (e.g., Ferguson, Hashtroudi \& Johnson 1992). Likewise, semantic similarity between the sources leads to more confusion as well (e.g., Lindsay, Johnson \& Kwon 1991). Against this backdrop of studies that have investigated the confusions that come from mistakenly judging average differences in qualitative characteristics is a small body of work that has argued that some source confusions can come from the retrieval of episodic memory traces of related memories. For example, Henkel and Franklin (1998) had people study visual line drawings of some objects and imagine drawings of other objects (also see Geraci \& Franklin, 2004). They found that people would often claim to have seen an object that they had only imagined if they happened to have studied a visually similar object, as compared with when they had not seen a similar-shaped object during the study sequence. Thus, seeing a lollipop will increase the probability of saying that one saw a magnifying glass that was actually only imagined. Note that the similarity in the Henkel and Franklin experiment (as in the present study) was at the item and not the source level.

Source-monitoring errors have also been studied in relation to false memories. False memories have commonly been examined using the Deese/Roediger-McDermott (DRM) paradigm. After studying a list of semantically related words (e.g., bed, pillow, rest), people often erroneously claim that a critical lure (e.g., sleep) was originally presented in the studied list (e.g., Gallo, 2006; Roediger \& McDermott, 1995). The high rates of false memories in the DRM paradigm are commonly thought to result from associative activation of the related lure that occurs during encoding or retrieval (Roediger, Balota, \& Watson, 2001). One dominant explanation for this kind of error derives from the source-monitoring work that has examined false memories (e.g., Anastasi, Rhodes, \& Burns, 2000; Hicks \& Hancock, 2002; Mather, Henkel, \& Johnson, 1997). Hicks and Hancock suggested that the representation of the related lure is activated at encoding and may be bound to the source characteristics of the studied associates, and that this subsequently leads to false source attributions at retrieval. Alternatively, Mather et al. suggested that people will confidently attribute a speaker to a critical lure in the DRM paradigm because other related memories become activated when considering the lure during testing, and the voice information from this related information is essentially imputed to the critical lure (i.e., the locus of the false memory occurs at retrieval). Consistent with this interpretation, Henkel and Franklin (1998) also found that people would call an imagined item "seen" if it came from the same semantic category as an item that had indeed been seen (e.g., shirt and pants). Likewise, Lyle and Johnson (2006) found that imagined items that were visually or conceptually similar to items that had been seen were more likely to be attributed a particular source characteristic of the seen item. Thus, an imagined lollipop or shirt was more likely to be associated with the corner of the computer screen (or the color of a picture) in which a magnifying glass or pair of pants had indeed been seen (see also Lyle \& Johnson, 2007). Lyle and Johnson (2006) argued that during testing the visually or conceptually similar object becomes reactivated and the features from the experienced object inadvertently become imported to the imagined object (Johnson \& Hirst, 1993).

In all of the cases studied to date (including the DRM paradigm), the source-monitoring error has been a confusion between internal and external sources, or a realitymonitoring error. We know of only one related study that has examined a similar error that occurred between two external sources. Hicks and Starns (2006) presented items from male and female sources or from seen and heard sources. In their critical manipulation, they presented at encoding, in addition to the target item, three primes that had forward association strength to a target item. For example, rocking, swivel, and desk all produce chair according to the Florida word association norms (Nelson, McEvoy, \& Schreiber, 1998) and were presented at encoding from either of two sources. The priming manipulation at encoding consisted of all three primes of a target word being presented in the same source as their target, all three primes being presented in a different source from their target, or no primes of a target word being presented at all. The source test included only studied target and filler items (i.e., no primes). Across three experiments, Hicks and Starns showed that when the source of the three primes was opposite to the source of the tested target word chair, source memory was significantly worse than for target words that had no studied primes. In addition, when the source of the three primes matched that of the tested target word, source memory was improved relative to the noprime condition (although this effect was only significant in Exp.2). Hicks and Starns's materials were designed such that there was no backward association strength from the tested items (e.g., chair) to the untested prime words (e.g., rocking, swivel, and desk). From the perspective of the importation or imputation theories described in the previous paragraph, Hicks and Starns tested the weakest-case scenario for finding an influence of related material being on the study list. According to those theories, had they tested on one of the prime words - say, rocking - the target word chair should have come to mind during the test, and with some probability, features of the related item may have become activated (or reactivated) and been imported or imputed to the target item, or otherwise have affected source memory for the target. Importation, in this instance, refers to the process of studied information affecting a 
source decision based on a related test item. Importantly, this reactivation effect occurs at retrieval, and the studied information that is being imported to the test item is not necessarily the exact same information from before, but instead is related in some manner. Thus, if rocking were a test item and were subsequently used to probe memory, the related source information from chair would import its source information to rocking and potentially affect the source decision. Note that this would be less likely to occur when chair is used as a test item, because chair is unlikely to reactivate rocking at retrieval.

The facilitation and decrement of accurate source memory in Hicks and Starns's (2006) report was achieved using three highly associative primes for each target word. Participants therefore had multiple pieces of source information that could potentially influence source responses. The purpose of the present study was to examine both forward and backward associates in order to investigate whether source attributes from a single item could influence source memory in a similar manner by using stimuli other than those from the DRM paradigm. By testing the influence of both forward and backward associates from a single study trial, we feel that our present manipulations build upon Hicks and Starns's previous work. It is important to look at backward associative strength because the conditions under which source memory biases operate in a bidirectional manner are still poorly understood. In our first experiment, we presented asymmetrical associates (i.e., word pairs) in either the same source or two different sources at encoding. For example, participants studied a series of words such as credit and card on separate trials intermingled throughout the study phase. The word credit we label the forward member of the pair because it produces the word card, which we label the backward member; obviously, this terminology is arbitrary. Analogously, the prime words used by Hicks and Starns (e.g., rocking, swivel, and desk) we consider forward members, whereas the target item (e.g., chair) we consider the backward member. The backward member does not produce the forward member(s) in free association norms. We predicted that when the forward member of the pair was tested, the source features of the backward member would be reactivated and potentially aid memory when the sources matched, or would decrease memory when the sources mismatched. When the backward member was tested, we did not expect the forward item to come to mind, because it was chosen to have a low association value to the forward item. In this manner, we are testing the importation theory using semantic associations in a paradigm using external sources, and specifically not using categorical associates. That is, encoded information from the backward member may be inadvertently reactivated upon presentation of the forward member at test, and this information may be imported to the forward member in consideration of its source. To foreshadow our other manipulations, we extended this line of reasoning to bidirectional associates as well as synonymous relations.

\section{Experiment 1}

In this first experiment, we are testing a theory of reactivating related episodic memory traces to determine whether source-monitoring processes make use of concurrently activated information. According to our reasoning, and Lyle and Johnson's (2006) theory, a forward member presented in the same source as the backward member should yield better source memory, as compared with when they occur in different sources.

\section{Method}

Participants Undergraduate students from the University of Georgia volunteered in exchange for partial credit toward a research appreciation requirement. Each participant was tested individually in sessions that lasted approximately $25 \mathrm{~min}$. A total of 37 volunteers were tested.

Materials and procedure We chose 28 pairs of words from the Florida word association norms (Nelson et al., 1998). The average association strength from the forward member to the backward member was .81 , whereas the strength in the opposite direction was .04. Thus, the forward member produced the backward member, but not vice versa. Examples of the stimuli used in Experiment 1 are provided in the first column of Table 1. The software controlling the experiment chose 14 pairs at random to be presented in the same source and 14 to be presented in different sources. For the pairs presented in the same source, half were presented visually on the computer monitor, and half were heard spoken auditorily. For the pairs presented in different sources, the software randomly chose half of the items to have the forward member spoken and the other half to have it seen visually. Consequently, of the 56 studied items, half were experienced in each source. Each item was studied individually, and the randomization procedure ensured that at least four trials separated the members of each pair during the study phase.

Table 1 Example stimuli across experiments

\begin{tabular}{lll}
\hline Experiment 1 & Experiment 2 & Experiment 3 \\
\hline Credit-Card & Salt-Pepper & Engine-Motor \\
Wick-Candle & Aunt-Uncle & Happy-Glad \\
Rye-Bread & Nail-Hammer & Vision-Sight \\
\hline
\end{tabular}


For each phase of the experiment, participants read instructions on the computer monitor that the experimenter also reiterated in her own words. During the study phase, some items were seen visually in the middle of the computer monitor for $3 \mathrm{~s}$ each. When the item was to be heard, the software wrote the word to the experimenter's workstation behind the participant, and she pronounced it out loud. Heard trials were also timed at $3 \mathrm{~s}$, but obviously the duration of speech for each item was much shorter than the trial duration. Participants had been instructed to study the items for an unspecified memory test. Upon conclusion of the study phase, a 4-min distractor task consisting of mathematical problems was administered. Following this, instructions for the test phase were given. Participants were asked to press a key marked "heard" or a key marked "seen" with one of their index fingers. We randomly intermingled 28 new items among the 56 studied items, and participants were asked to press the space bar with one of their thumbs for these new items. The test phase was self-paced. We note that all randomization during study and test was done without regard to whether forward members were presented before or after their backward members, and vice versa. While the presentation at test was random, the directional word pairs were separated by an average distance of about 28 trials. This average separation was consistent across all reported experiments.

\section{Results and discussion}

Unless otherwise noted with a specific $p$ value, all statistical tests are significant at the conventional $5 \%$ probability of a Type I error. Before reporting the results, we deal first with several data-analytic issues that apply to this and the subsequent experiments. In many source-monitoring experiments, it is customary to report recognition hit rates that sum the correct attributions with the misattributions to the other source for the studied items. That is, we calculated the proportion of times participants correctly recognized a studied item as "old" by choosing either source, regardless of the actual source accuracy (see, e.g., Hicks \& Cockman, 2003). In Experiments 1 and 2, recognition hit rates hovered near 82\%, whereas in Experiment 3 the recognition hit rate was $73 \%$. In none of these cases were there any significant differences among the various conditions within an experiment, and moreover, the false alarm rates were very low, averaging $3.9 \%$ and $5.3 \%$ for the seen and the heard sources, respectively. The absence of differences in recognition hit rates and the lack of bias to choose one source over another allow us to report average conditionalized source identification measures (ACSIM) as the metric of source-monitoring performance. This metric is calculated as the correct source attributions divided by the recognition hit rate, averaged across both sources. Essentially, this measure reports the percentage of the old items identified as "old" (i.e., recognized) that were correctly attributed to their studied source.

For this experiment, the ACSIM scores are displayed in Fig. 1. The left-hand and right-hand sets of bars represent pairs presented in the same source and different sources, respectively. The darker bars denote performance for the forward member, whereas the lighter bars denote performance on the backward member. We conducted a 2 (same vs. different source) $\times 2$ (forward vs. backward member) ANOVA with repeated measures on both factors. The main effect of same versus different was significant, $F(1,36)=$ $4.58, p=.039, \eta_{p}^{2}=.11$, as was the main effect of forward versus backward, $F(1,36)=14.8, p<.001, \eta_{p}{ }^{2}=.29$. These main effects were qualified by a significant interaction, $F(1$, 36) $=5.17, p=.029, \eta_{p}{ }^{2}=.13$. As the reader can see, for the forward member, source monitoring was better when members of the pair were presented in the same source rather than in different sources, $t(36)=3.38, p=.002, d=0.79$. However, there was no difference in source-monitoring performance for the backward members, $t(36)=0.15$, $p=.88$. Therefore, these data are consistent with the notion that when the forward member is tested, the association to the backward member causes it to be reactivated at test. With these source characteristics activated, this information then influences performance on the forward member. As can be seen in the left-hand bars in Fig. 1, source memory for the forward member is facilitated when its associate is presented in the same source. However, when the source of the associate differs, performance on the forward member is equivalent to that for both types of backward members. In terms of the earlier example, when the forward member credit is presented at test, it activates the associated word card. The reactivation of source information for card then

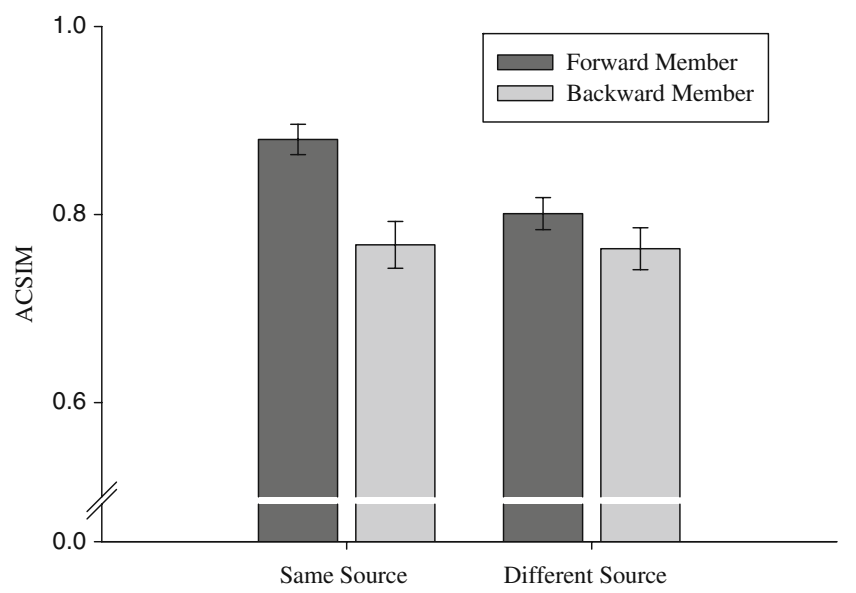

Fig. 1 Average conditionalized source identification measures of Experiment 1 
influences the source decision on credit. One could assume that the reverse influence is absent, although Hicks and Starns's (2006) report might argue otherwise. If this assumption is made, the data suggest that the primary influence on the forward member is to increase source memory when both items are presented in the same source, rather than performance being hurt when the pair members are presented in different sources. However, we acknowledge that this claim is somewhat speculative at this juncture.

\section{Experiment 2}

The results from Experiment 1 demonstrated that a single associated item on the study list can influence sourcemonitoring performance. Recall that Hicks and Starns's (2006) influence required the presentation of three associates in the same direction, toward what we have labeled the backward member, prior to testing on the backward member. Thus, a single forward association seems to have a sizable effect on source memory. In this next experiment, we theorized that items having a strong bidirectional association would also influence each other at test. Thus, with pairs like salt-pepper and aunt-uncle, either member of the pair should reactivate the other member during testing and should influence source monitoring. Obviously, there is no practical distinction to be made between forward and backward members in this experiment, because both members are essentially forward. Consequently, we predicted that presenting pair members in the same source would result in better source memory than presenting them in different sources.

\section{Method}

Participants A group of 37 new volunteers were recruited from the University of Georgia undergraduate student population. They received credit toward a research appreciation requirement. Two participants were replaced for essentially pressing the same response key throughout the entire test phase.

Materials and procedure A new set of 28 pairs of items were obtained from the Florida word association norms (Nelson et al., 1998). Each member of the pair had a strong bidirectional association to the other member, and as in the examples given earlier, each, when given as a cue, produced the other as a strong target item in the norms. Examples of the stimuli used in Experiment 2 are provided in the second column of Table 1. We used the same software as in Experiment 1 and arbitrarily assigned one member of the pair as the forward and the other as the backward associate. The average association value from the forward to the backward member was .65 , and the reverse association value was .60 . The randomization procedures were identical to those in Experiment 1, but pair membership was ignored for this experiment. In all other respects, this experiment was identical to the previous one.

\section{Results and discussion}

The ACSIM data are displayed as the left-hand pair of bars in Fig. 2, with the darker bar denoting performance for pair members presented in the same source. We conducted a 2 (same vs. different source) $\times 2$ (forward vs. backward member) ANOVA with repeated measures on both factors. As we predicted, source monitoring was better when members were presented in the same as compared with different sources, $F(1,36)=18.52, p<.001, \eta_{p}{ }^{2}=.34$. Neither the main effect of forward versus backward, $F(1,36)=0.03, p=.863$, nor the interaction, $F(1,36)=$ $0.11,=.748$, was statistically significant. The reader should recall that members of the pairs were neither studied together nor tested together. Therefore, this influence on source accuracy is most likely a consequence of the tested item reactivating its associated member, and the activated source characteristics then influencing the judgment on that test trial (Lyle \& Johnson, 2006, 2007).

\section{Experiment 3}

The first two experiments examined the reactivation of an item associated with the target via preexperimental learning, with the conclusion that this reactivation affects source monitoring. In this last experiment, we took the opportunity

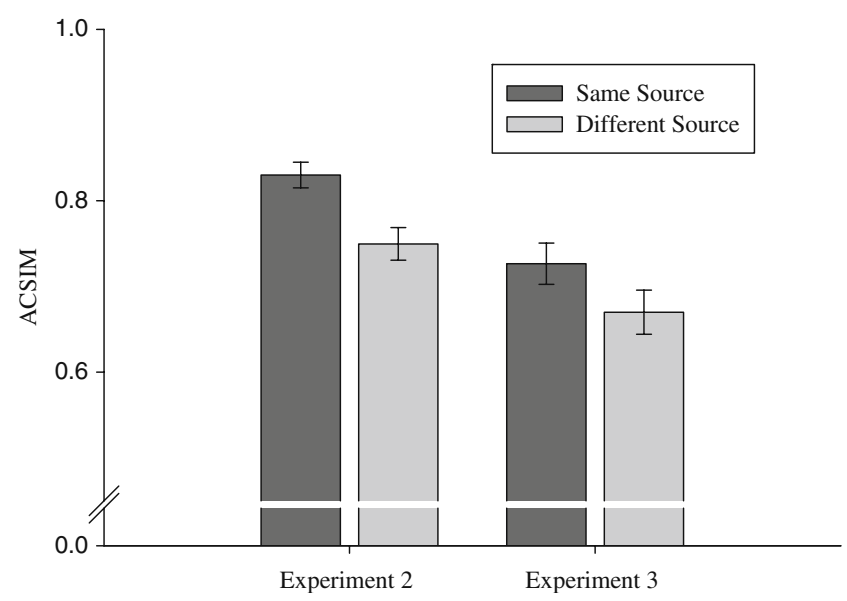

Fig. 2 Average conditionalized source identification measures for Experiments 2 and 3 
to study whether synonymy would operate in a fashion analogous to the associations studied in Experiment 2 . Synonyms are not uniformly generated as associates in the Florida word association norms. Nevertheless, they share a conceptual relationship and may serve to reactivate one another in a source test. Therefore, we wanted to ascertain whether studying happy or engine would reactivate a studied synonym (e.g., glad or motor) and affect source performance in the same way as in Experiments 1 and 2. Geraci and Franklin (2004) found that the highest rate of falsely claiming to see a picture that had only been imagined occurred when a picture of a synonymous item had been seen (e.g., an oar and a paddle). That outcome suggests that despite their low association values in production norms, synonyms can influence one another in a source test.

\section{Method}

Participants The 37 volunteers for this experiment were undergraduate students at the University of Georgia. They all received partial credit toward a research participation requirement, and were tested individually in sessions lasting approximately $25 \mathrm{~min}$.

Materials and procedure A set was assembled of 28 synonym pairs that had low association values in the Florida word association norms (Nelson et al., 1998). The same software that was used in Experiment 1 was used again. Therefore, synonym pairs were arbitrarily designated as forward and backward members. In so doing, the forward-to-backward association value was .19, and the reverse value was .14. Just as in Experiment 2 with the bidirectional stimuli, the forward-versus-backward distinction held no meaning. Examples of the stimuli used in Experiment 3 are provided in the third column of Table 1. As in Experiments 1 and 2, we used seen versus heard sources. The new items were not synonyms of the studied items, but they were control-matched to the studied items on characteristics such as frequency, syllable count, and length. As in Experiment 2, pair membership was ignored for the randomization procedure during both study and test. In all other respects, this experiment was conducted identically to Experiment 1.

\section{Results and discussion}

The results are summarized in the right-hand pair of bars in Fig. 2, with the darker bar denoting ACSIM performance when the synonyms were presented from the same source.
Again, we conducted a 2 (same vs. different source) $\times 2$ (forward vs. backward member) ANOVA with repeated measures on both factors. The pattern of results was identical to that found in Experiment 2. When an item was tested, source memory was better if the synonym pair had been studied in the same source as compared with when its members were presented in different sources, $F(1$, 36) $=4.7, p=.037, \eta_{p}{ }^{2}=.12$. This outcome suggests that there may be alternative mechanisms for reactivating other studied items, such that semantically related items can influence source memory in a manner similar to a direct preexperimental association. Neither the main effect of forward versus backward, $F(1,36)=0.03, p=.862$, nor the interaction, $F(1,36)=0.62, p=.435$, was statistically significant. As noted earlier, the recognition hit rate was the lowest of all the experiments, so participants obviously had some difficulty discriminating targets from lures. Nonetheless, the important point is that synonyms serve the same function of reactivating related list members, and when they are studied in the same source, this serves to improve source memory.

\section{General discussion}

These experiments were inspired by work showing that people more often will falsely claim to have seen something that was only imagined when they have actually seen a physically similar or conceptually related (by category) item (Henkel \& Franklin, 1998). When people make these errors, they also tend to import or borrow features from the experienced items, such as their location on the computer monitor or their color (Lyle \& Johnson, 2006, 2007). The experiments in this study demonstrate a related phenomenon, namely that backward associates to an item can affect source memory. We also found that, in addition to associations, synonymy affected source monitoring. Our preferred explanation for these effects is that a test item's power to evoke related information from the study list determines whether that related information will affect performance. Reactivating associated material appears to influence source monitoring.

We did not find evidence, as Hicks and Starns (2006) did, that test performance on the backward member of a pair was influenced by the source of the forward member. Of course, Hicks and Starns used three associates, and we used only one. Their results were discussed in terms of global matching models, in which the target item probe, containing both item and source information, is matched to the features of all stored memory traces to produce an activation value. Thus, when multiple forward associates are studied, each individual associate creates its own activation at test, and these values are summed into the 
global match strength in order to make a source decision. Similarly, the source-monitoring framework predicts that source errors occur when there is a high degree of overlap in source characteristics. When considering a target's source, features of a related item may inadvertently be reactivated and imported to the candidate memory and may erroneously be used as evidence that a target originated from a certain source. Perhaps the reactivation of only one forward associate in the present study was insufficient to obtain the effects reported by Hicks and Starns, suggesting that the effects of forward and backward associates on reactivation of features work in different manners. That is, backward associates may require multiple pieces of associated information to be reactivated, in order to influence source memory.

We also favor a retrieval explanation of the present findings. We believe that when a test item is presented and used to probe memory, other related information from encoding is reactivated and may affect the source decision on the target item (i.e., importation). If this were based on an associative encoding influence, we would predict a stronger effect on the backward member of the associated pair in Experiment 1. For example, when credit (the forward member) is studied, card (the backward member) should also come to mind. If this encoding association accounted for the results, we should have found a stronger effect on the backward member at test. However, a retrieval mechanism would predict a stronger effect on the forward member, because of the effect of the backward member on the test decision. When card is presented at retrieval, it is much less likely to elicit credit, as compared to the other way around. Even so, these results do not exclude the possibility of an encoding influence, and manipulations such as those done by Hicks and Starns could produce a stronger influence of encoding processing. Future work is needed to further explore these different accounts.

In Experiments 2 and 3, we do not know whether the related item being studied in the same source improved performance, or whether studying it in another source hurt performance (or some combination of both). However, Lyle and Johnson (2007) found that when imagined objects were correctly recognized as old, location judgments for these items were more accurate when visually similar objects had been seen in the same location at study, as compared to when control pairs with no similarity were presented. When seen objects were presented in a different location than visually similar imagined objects, location accuracy for the imagined objects did not differ reliably from accuracy for the control pairs. This suggests that the importation of congruent source information serves to enhance memory performance, whereas incongruent source information does not necessarily hurt performance.
In Experiment 1, however, a stronger case could be made that the importation of source information serves to improve memory when the sources of the pair members match. Using a no-prime condition as a baseline measure, Hicks and Starns (2006) found that forward associates presented in a different source consistently provided a more sizable decrement to source memory for backward members than the benefit they provided for forward associates studied in the same source. Although we did not include a control measure in our study, we do not feel that this takes away from the significance of our results. We predicted that reactivated features from the backward member would influence performance for the forward member. As we argued earlier, when testing on the backward member of a pair, there should be no strong reason for the forward member of the pair to come to mind. For example, the first several associates for card are game, ace, deck, poker, and birthday, and the word credit is virtually never given as an associate. As can be seen in Fig. 1, the effect seems to be in the direction of the related information improving source memory, because the source memory performance on the forward member when the sources differ is equivalent to the performance on backward members when they are presented either in the same or in difference sources. Thus, using the backward items as a form of control item, the direction appears to be toward improving source memory. Therefore, using bidirectional pairs in Experiments 2 and 3, we did not feel it was necessary to include control items, because there was no influence from the forward member at test.

Although the results from these experiments suggest that same-source items appear to improve memory performance, we do not wish to make a strong case for this stance, because it is based on an assumption that could be wrong. In addition, it is unclear to us why the effect would only work to improve memory and not to decrease it when the source information is inconsistent across members of a pair. One possibility is that source information of items with high forward associations can be bound in memory with related information. The encoding of a word can correspond to the simultaneous activation of a set of features (which could include features common to related items). Therefore, mistakenly reactivating feature information of the related member at test serves to facilitate memory performance when the source information is congruent (e.g., both items were seen) because participants may disregard the features that distinguish the two items (Anisfeld \& Knapp, 1968). However, when the two members are encoded in different sources (e.g., seen and heard), sensory information for associated items may be better distinguished and more strongly bound to its appropriate target. Although some common features of related items may be reactivated at test, source information from the backward member may not be 
reactivated upon consideration of the forward member's source, thus producing no benefit to memory when the sources differ. An alternative explanation could be that people only search for corroborating evidence for weakly learned items. A familiarity-plus-corroboration account would suggest that high levels of familiarity cause participants to search their memory for corroborating details that are borrowed from related items and bound to a false memory trace (Lampinen, Neuschatz, \& Payne, 1999). Thus, the evidence from the reactivated item is only used to push one toward an already tentatively chosen candidate decision. We readily admit that this theory is offered in a post-hoc manner.

One alternative explanation for the present results is that, rather than increasing source memory for the target item, the backward member may merely set up a response bias for that trial. This idea is consistent with the previous account that people may be influenced by same-source information only when the item-source association is weakly bound. In these weak-encoding scenarios, participants may be more influenced by response bias. While the data do not speak to whether or not people consciously search for related information from the study phase, Lyle and Johnson $(2006,2007)$ found that most participants were unaware of the fact that they had studied physically and conceptually similar items. Although we did not query our participants, we suspect that they did not notice the relations, and therefore we do not believe that participants explicitly accessed related item information when making source decisions. Consequently, bias should not influence results without memorial access to the related item. Rather, we believe that reactivation of partial information from the related item influences performance primarily in an implicit manner. In addition, it is not evident that response bias would only increase performance in the same-source situations, without decreasing source memory in the different-source scenarios. Future work is needed to further delineate and contrast these partial-information and response-bias accounts.

Thus, in everyday source monitoring, it is probable that an analogous phenomenon occurs relatively automatically. For example, if one is trying to remember the person who imparted a piece of information, such as that interest rates will be rising, one might have a higher probability of specifying the correct source if one had a conversation about banks and/or loans with the same person. We believe that this occurs because memory traces are essentially bundles of features (see, e.g., Bower, 1967). Sourcemonitoring processes can make use of bits and pieces of these bundles, and in addition, partial and incomplete information can be successfully assembled to arrive at a correct source decision (Dodson, Holland, \& Shimamura, 1998; Gruppuso, Lindsay, \& Kelley, 1997; Hicks, Marsh, \&
Ritschel, 2002). Thus, when related information becomes reactivated, the bits and pieces of its bundle can commingle with the already activated information from the test candidate. The results from this study argue quite strongly for such a theory, because the errors observed here cannot be easily derived from a theory in which a source is recollected in an all-or-none fashion.

This argument is also consistent with some of our previous work on source monitoring (Cook, Marsh, \& Hicks, 2006), as are the outcomes of this study. When people learn cue-target paired associates, we wanted to ascertain whether source information about the target was available in the absence of being able to recall that target when presented with the cue. The goal was to understand whether partial source information potentially contributes to the information that fuels feeling-of-knowing judgments or that lead people to tip-of-the-tongue states (e.g., Schwartz, 1994, 2002). The answer to our question was complex, insofar as these partial source details were not accessible when learning was poor (i.e., a single study trial). However, when learning was good (three study trials per pair) or learning was intentional, participants were able to recover some source information even though they could not recall the target item itself. The same thing appeared to happen in this study, because a strong forward association to another studied item affected source memory, but the backward member did not appear to be affected. These outcomes suggest that access to bits and pieces of partial source information is only available when there is a strong association between the two pieces of information, consistent with the reactivation hypothesis. Such an association was present with the preexperimental associations we used here.

The present study highlights that source-monitoring processes make use of activated information. That information comes not only from the candidate memory, but also results from how the test cue evokes information from memory. Sometimes the cue to a source decision is incomplete or misleading, in which case the activated information may be insufficient to evoke anything but a vague feeling of familiarity. In other cases, as with the present study, additional information can be activated or reactivated and may inadvertently influence a source judgment. We are unclear as to just how successful applying more stringent decision processes would be at reducing this influence. Information that comes to mind automatically is often hard to inhibit if one is not even aware that it is influencing performance. In addition, as depicted in the sourcemonitoring framework, most source decisions are made rather quickly and heuristically on the basis of a comparison of activated records to some expected average that is typical of items from a candidate source. Partial information from an associated or conceptually related memory trace probably 
goes undetected in these cases. Our point is that it may be very difficult to detect and reduce the influence that contextual information from related material has on a source judgment. To date, the studies that have examined the influence of related materials from the study phase have been more concerned with demonstrating that an influence exists. We are guilty of such a focus, too, but perhaps a fruitful avenue for further exploration will concern the decision criteria that can be adopted (perhaps based on knowledge of the situation) that might reduce or eliminate this effect of related episodic information on a source-monitoring judgment.

\section{References}

Anastasi, J. S., Rhodes, M. G., \& Burns, M. C. (2000). Distinguishing between memory illusions and actual memories using phenomenological measurements and explicit warnings. The American Journal of Psychology, 113, 1-24.

Anisfeld, M., \& Knapp, M. (1968). Association, synonymity, and directionality in false recognition. Journal of Experimental Psychology, 77, 171-179.

Bower, G. H. (1967). A multi-component theory of the memory trace. In K. W. Spence \& J. T. Spence (Eds.), The psychology of learning and motivation (Vol. 1, pp. 229-325). New York: Academic Press.

Cook, G. I., Marsh, R. L., \& Hicks, J. L. (2006). Source memory in the absence of successful cued recall. Journal of Experimental Psychology. Learning, Memory, and Cognition, 32, 828-835.

Dodson, C. S., Holland, P. W., \& Shimamura, A. P. (1998). On the recollection of specific- and partial-source information. Journal of Experimental Psychology. Learning, Memory, and Cognition, 24, 1121-1136.

Ferguson, S. A., Hashtroudi, S., \& Johnson, M. K. (1992). Age differences in using source-relevant cues. Psychology and Aging, $7,443-452$.

Gallo, D. (2006). Associative illusions of memory: False memory research in DRM and related tasks. New York: Psychology Press.

Geraci, L., \& Franklin, N. (2004). The influence of linguistic labels on source monitoring decisions. Memory, 12, 571-585.

Gruppuso, V., Lindsay, D. S., \& Kelley, C. M. (1997). The processdissociation procedure and similarity: Defining and estimating recollection and familiarity in recognition memory. Journal of Experimental Psychology. Learning, Memory, and Cognition, 23, 259-278.

Henkel, L. A., \& Franklin, N. (1998). Reality monitoring of physically similar and conceptually related objects. Memory \& Cognition, 26, 659-673.

Hicks, J., \& Cockman, D. (2003). The effect of general knowledge on source memory and decision processes. Journal of Memory and Language, 48, 489-501.

Hicks, J. L., \& Hancock, T. W. (2002). Backward associative strength determines source attributions given to false memories. Psychonomic Bulletin \& Review, 9, 807-815.

Hicks, J. L., Marsh, R. L., \& Ritschel, L. (2002). The role of recollection and partial information in source monitoring.
Journal of Experimental Psychology. Learning, Memory, and Cognition, 28, 503-508.

Hicks, J. L., \& Starns, J. J. (2006). Remembering source evidence from associatively related items: Explanations from a global matching model. Journal of Experimental Psychology. Learning, Memory, and Cognition, 32, 1164-1173.

Johnson, M. K., Foley, M. A., \& Leach, K. (1988). The consequences for memory of imagining in another person's voice. Memory \& Cognition, 16, 337-342.

Johnson, M. K., Foley, M. A., Suengas, A. G., \& Raye, C. L. (1988). Phenomenal characteristics of memory for perceived and imagined autobiographical events. Journal of Experimental Psychology: General, 117, 371-376.

Johnson, M. K., Hashtroudi, S., \& Lindsay, D. S. (1993). Source monitoring. Psychological Bulletin, 114, 3-28.

Johnson, M. K., \& Hirst, W. (1993). MEM: Memory subsystems as processes. In A. F. Collins, S. E. Gathercole, M. A. Conway, \& P. E. Morris (Eds.), Theories of memory (pp. 241-286). Hove: Erlbaum

Johnson, M. K., \& Raye, C. L. (1981). Reality monitoring. Psychological Review, 88, 67-85.

Lampinen, J., Neuschatz, J., \& Payne, D. (1999). Source attributions and false memories: A test of the demand characteristics account. Psychonomic Bulletin \& Review, 6, 130-135.

Lindsay, D. S., Johnson, M. K., \& Kwon, P. (1991). Developmental changes in source monitoring. Journal of Experimental Child Psychology, 52, 297-318.

Lyle, K. B., \& Johnson, M. K. (2006). Importing perceived features into false memories. Memory, 14, 197-213.

Lyle, K., \& Johnson, M. (2007). Source misattributions may increase the accuracy of source judgments. Memory \& Cognition, 35, $1024-1033$.

Mather, M., Henkel, L. A., \& Johnson, M. K. (1997). Evaluating characteristics of false memories: Remember/know judgments and memory characteristics questionnaire compared. Memory \& Cognition, 25, 826-837.

Mitchell, K. J., \& Johnson, M. K. (2000). Source monitoring: Attributing mental experiences. In E. Tulving \& F. I. M. Craik (Eds.), The Oxford handbook of memory (pp. 179-195). Oxford: Oxford University Press.

Mitchell, K. J., \& Johnson, M. K. (2009). Source monitoring 15 years later: What have we learned from fMRI about the neural mechanisms of source memory? Psychological Bulletin, 135, 638-677.

Nelson, D. L., McEvoy, C. L., \& Schreiber, T. A. (1998). The University of South Florida word association, rhyme, and word fragment norms. Available at www.usf.edu/FreeAssociation/.

Roediger, H. L., III, Balota, D. A., \& Watson, J. M. (2001). Spreading activation and arousal of false memories. In H. L. Roediger III, J. S. Nairne, I. Neath, \& A. M. Surprenant (Eds.), The nature of remembering: Essays in honor of Robert G. Crowder (pp. 95115). Washington: American Psychological Association.

Roediger, H. L., III, \& McDermott, K. (1995). Creating false memories: Remembering words not presented in lists. Journal of Experimental Psychology. Learning, Memory, and Cognition, $21,803-814$.

Schwartz, B. L. (1994). Sources of information in metamemory: Judgments of learning and feelings of knowing. Psychonomic Bulletin \& Review, 1, 357-375.

Schwartz, B. L. (2002). Tip-of-the-tongue states: Phenomenology, mechanism, and lexical retrieval. Mahwah: Erlbaum. 